Journal of Mathematics and Statistics 7 (4): 262-269, 2011

ISSN 1549-3644

(C) 2011 Science Publications

\title{
The Importance of Numbers and the Need to Study Primes: The Prime Questions
}

\author{
Matthew Curtis and Gurudeo Anand Tularam \\ Department of Mathematics and Statistics, \\ Faculty of Science Environment Engineering and Technology, \\ Centre of Environmental Futures, Griffith University Brisbane, Australia
}

\begin{abstract}
Problem statement: The study of prime numbers is important today as it was in ancient times. The level of interest in any form of mathematics of students in Australian high schools is decreasing and furthermore the level of interest in number theory is also less that it was before; prime numbers is not being considered as an area of interest by our younger stars. This paper explores prime numbers and attempts to show some interesting aspects in the study of primes that highlight why number theory studies can be considered important for today's up and coming mathematicians. Approach: The historical review of mathematics and analysis of simpler but accessible proofs can help show clearly the motivations behind the mathematicians work. In depth analysis work may be used to reveal parts passion, politics and dedication played in the study of numbers and construction of equations. The analysis of proofs is also used to provide a further background of prime numbers and why the pursuit of primes. Results: The analysis shows there are a number of motivating factors that may promote real interest in number theory; such as human dramas behind the "prime" mathematics. The paper shows politics and passion also exist behind numbers. If explicated well, this may help drive more interest in research by younger mathematicians by making mathematics human activity. In number theory there are a number of unanswered questions that often attract large sums of money. There are a number of proofs in primes and these are made accessible to the younger researchers so that they appreciate the nature of the problems faced by the history's intellectual minds in their efforts to solve some of the unanswered questions; and those they began to solve. The analysis based on historical works of mathematicians as well as the modern studies reveal surprising "real life" applications for prime number theory. Conclusion: Mathematics can often be thought as only for the enjoyment of pure thought but the study of prime numbers has discovered to have some real life applications. This paper shows that pure study of abstract ideas, importance of numbers, history, politics and real life applications are all important aspects of the study of prime numbers. If these aspects are highlighted, a more realistic picture of the pure mathematics of prime numbers may be presented in classrooms to the younger up and coming mathematicians of today to help promote interest to pursue research work on prime numbers. Allowing for different points of view to the study of mathematics should be well regarded in classrooms that my in itself lead to better understanding of the very building blocks of our numbers system.
\end{abstract}

Key words: Prime numbers, mathematical proofs, prime questions, equation converges, natural numbers, finite number, study primes, fundamental theorem, arithmetic states, mathematical proof

\section{INTRODUCTION}

The history of prime numbers is an extensively covered field by modern and historical researchers alike but few authors provide a succinct comprehensive review of the development over time in a manner accessible to most. This study attempts to provide such a review as well as examine several interesting unanswered topics in number theory (Al Mijalli, 2011; Ribenboim, 1996). The definition of a prime is rather simple but the problems (headaches) its investigation has given to number theorists have been enormous. It is clear that natural numbers were to first to appear with other categories shortly following as the need developed for

Corresponding Author: Gurudeo Anand Tularam, Department of Mathematics and Statistics, Faculty of Science Environment Engineering and Technology, Centre of Environmental Futures, Griffith University Brisbane, Australia 
them increased such as "owing" may have led to negative integers and so on. A prime number is simply a natural number that is only divisible by 1 and itself. All other integers are known as composite numbers. The first ten prime numbers can be easily listed: $2,3,5,7,11,13$, $17,19,23$ and 29; interestingly, only the number two is an even prime number. In the literature, there are many papers that cover specific topics extensively but there is a paucity of interesting overviews of the simplicity of early proofs, questions asked, answered and unsolved in the literature for younger mathematicians to explore. Little is known about what the real life aspects behind the numbers represents. There is also not much written on the passion, politics and real life but rather one notes instead numerous texts on higher level work with pages of lifeless numbers. This approach does not sell the interesting topic of prime numbers to the public or present motivating topics for younger minds to research particularly when they meet prime numbers in earlier years at high school for example. An area where there are still a number of interesting questions unanswered. The topic of prime numbers needs the attention of the largest collection of questioning younger minds. Century old proofs such as Euclid's proof of infinite prime numbers and unanswered questions like Riemann's Conjecture are interesting to the passionate mathematics adventurer but to solve the many questions that have remained unbeaten for hundreds of years there is a need to generate new interest at the younger level so that a multitude of interested observers may help undertake the task. This study hopes to give an interesting and more accessible explanation to young mathematicians by giving a real life historical recount of the 'prime' questions and the topic of prime numbers to create greater passion for the topic in high schools for example.

The lower numbers of students taking up mathematics is noted in recent times and this study may help renew interest in pure mathematics and may inspire leaps in passion that can only help progress further understanding of the pure building block of mathematics. Presently, finding the biggest prime number is a major goal for all but for new mathematicians who are discovering and learning about the topic of prime numbers need to know and understand why primes are fascinating. The aim of this study is to increase interest in pre mathematics at the high school level by showing how prime numbers leads to an appealing and remarkable area of pure mathematics study.

A brief historical perspective: Humans have studied many questions over time such as those related to mathematics yet why should the study of numbers and their properties be so important to us is not completely obvious to all. The human history now shows that all ancient cultures had a concept and use for numbers. There cultures ranged from the primitive tribes who counted to up to three to the Hindus, Chinese, Mayan and Aztec, Egyptians and Roman's including others all appear to use symbols or numerals of sorts to represent numerical quantities in recordings. The present day decimals can be attributed to the ancient Hindus including the use of the zero. The Hindus were the first to use zero as a place value and used the decimal system that we study today. In fact, the circle method used in modern study of primes may be attributed to the Indian methods generating when solving the so called Diophantine equations.

Anyone who needed to count a group of objects whether as complicated as counting the stars or more simply the number of toes a person has required some sort of numbering. Any counting requiring more use then the ten fingers and toes of the one doing the counting needed a way to group numbers together to create a sy stematic way to reach larger numbers. The basic decimal and decadic group uses groups of 10 and is widely used around the world today. Throughout history cultures have used their own systematic way to group numbers; Babylonian sexagesimals system which used the basic grouping of 60 which can be reflected when measuring time and angles. Hindu decimal system is another where simply body parts such as fingers and toes led to the use of 10 as a somewhat simpler system to use. The historical development of numbers and the proceeding human fascination with different bases alone is a strong enough motivation and reason to study the properties of numbers. There needs to be some understanding for the systems used that functioned efficiently and then to develop, evolve and adapt to modern demands. As a culture that has an immense growing demand on numbers, there is a need improve continuously our understanding of the numbers we utilize every day (Ore, 1988; Al Mijalli, 2011).

Number theory is the division of pure mathematics concerned with the properties and interactions of numbers. A traditional mathematician is interested in finding logic, patterns and puzzles through the better understanding of numbers and there functionality. The field of number theory appears to have all the enticements a mathematician needs to spend a life time of passionate researching in the area of pure numbers. In fact many of history's greatest minds have all shared an interest in number theory and specifically the study of prime numbers. For example Euclid, Riemann, Goldbach and Euler are just a few who researched this field for many years and together provided proofs and 


\section{J. Math. \& Stat., 7 (4): 262-269, 2011}

posed conjectures in the field of prime numbers so the mathematicians who follow them can stand on the shoulders of such giants and begin their own search.

Definitions: While a good mathematical proof is required for the understanding of mathematics at its fundamental level, it is no good for any aspiring mathematician who is for the first time looking at how and why prime numbers function the way they do. To remedy this there will be a simplified quick explanation of any mathematical proofs in advance to help educate instead of prove the fundamental points of prime numbers.

A prime number is an integer greater than one that has exactly two positive divisors, the number itself and 1. The numbers are then divided into two sets a prime set and a composite set. A natural integer that has a finite number of divisors greater than two is known as a composite number. There are other important words commonly used in the work on prime numbers such as a common divisor. A common divisor is a number that divides at least two other integers' simultaneously. The greatest common divisor ( $g c d$ ) is the largest common divisor shared by some other integers.

The Fundamental Theorem of Arithmetic states that any given integer can be reduced to a unique set of prime divisors. This can be revealed from a few uncomplicated facts about integers and prime numbers. An integer (with a prime divisor $\mathrm{p}$ ) can be represented by its composite divisors. (If it only possesses divisors that are primes then the fundamental theorem holds true) From this it can be shown that one of its composite divisors is also divisible by that same prime number the integer is. It is then easy to show that what are left are either a prime and a composite number or a prime and two composite numbers. The composite number or numbers can continue to be broken down in this fashion until all the divisors of the integer are primes. The final list of prime numbers is unique to the original integer and cannot correspond with a different representation of primes. After knowing just this fact about the prime numbers it becomes clear as to why primes are so important and intriguing. They are indeed the building blocks of every number. To understand them would be to understand numbers and the most basic level.

The proof is as follows and is provided for a more detailed view of the pure mathematics behind prime numbers.

If $\mathrm{p}$ is any prime number then we write $\mathrm{p} \mid \mathrm{ab}$ where $a$ and $b$ are positive integers to mean $p \mid a$ or $p \mid$ b. Showing this to be the case we reject the provision where $\mathrm{p} \mid \mathrm{a}$ (or it shows the above statement to be true) and only looking at the case where $\mathrm{p}$ a then it can be shown that the $\operatorname{gcd}(\mathrm{p}, \mathrm{a})=1(\operatorname{from} \operatorname{gcd}(\mathrm{p}, \mathrm{a})=\mathrm{p}$ or 1 where $\mathrm{p} \mid \mathrm{a})$. This is the case because $\mathrm{p}$ is prime with only itself and one as its divisors and so sets the condition on the gcd. Therefore $\mathrm{p} \mid \mathrm{b}$. Generalisation the above statement exemplifies that if $p \mid a_{1} a_{2} a_{3 \ldots} a_{n}$ then $\mathrm{p} \mid \mathrm{a}_{\mathrm{m}}$ where $1 \leq \mathrm{m} \leq \mathrm{n}$. Starting from the point $\mathrm{n}>$ 2 because for $n \leq 2$ the proof holds true from the first explanation where $\mathrm{p} \mid \mathrm{ab}$ for $\left(\mathrm{a}=\mathrm{a}_{1}, \mathrm{~b}=\mathrm{a}_{2}\right.$ Then $\mathrm{p} \mid \mathrm{a}_{1}$ $a_{2} a_{3} \ldots a_{n-1}$ or $p \mid a_{n}$ if it is the latter then the first statement holds true and if it's the former then through induction it stands that $\mathrm{p} \mid \mathrm{a}_{\mathrm{m}}$ where $1 \leq \mathrm{m} \leq \mathrm{n}-1$. For either case $p$ is the divisor for one of the numbers $a_{1} a_{2}$ $a_{3 \ldots} a_{n}$. For the special case where $a_{1} a_{2} a_{3} \ldots a_{n}$ are all primes then $\mathrm{p}=\mathrm{a}_{\mathrm{m}}$ given that $\mathrm{a}_{\mathrm{m}}$ from the definition of $\mathrm{a}$ prime number is only devisable by itself and 1 . Providing the conclusion that $\mathrm{p}=\mathrm{a}_{\mathrm{m}}$ (from $\left.\mathrm{p} \mid \mathrm{a}_{\mathrm{m}}\right)$, $(\mathrm{p}>$ 1). These simple definitions of integers and prime numbers pave the way to one of the most important fundamental theorems in number theory.

Fundamental Theorem of Arithmetic states that any natural number greater than one can be represented by a unique product of prime numbers. Knowing that $\mathrm{n}=\mathrm{ab}$ and $\mathrm{p} \mid \mathrm{ab}$ then $\mathrm{a}, \mathrm{b}=\mathrm{p}_{1} \mathrm{c}\left(\right.$ or $=\mathrm{p}_{1}$ or $\left.=\mathrm{p}_{1} \mathrm{p}_{2}\right)$ for the non trivial case $a$ and $b$ can now be represented $n=p_{1} b c$ or $\mathrm{n}=\mathrm{acp}_{1}$ Following this course of action until all divisors of the newly formed composite integers are primes it can be seen that $n=p_{1} p_{2} \ldots p_{m}$ and therefore broken into its prime divisors. A case where $\mathrm{n}$ does not share the uniqueness of the products of primes would be evidence for $n=p_{1} p_{2} \ldots p_{m}=q_{1} q_{2} \ldots q_{k}(m \leq k)$ where there are at least two sets of differing prime products ( $p$ and $q)$. This being the case where $p_{i} \mid q_{j}$ and both are primes it is recognized that $\mathrm{p}_{\mathrm{i}}=\mathrm{q}_{\mathrm{j}}(\therefore \operatorname{gdc}(\mathrm{p}, \mathrm{q})=(\mathrm{p}$ or $\mathrm{q}), 1$; as explained above). This is also the case for some $\mathrm{q}_{\mathrm{j}}=\mathrm{p}_{\mathrm{i}}$. The set of primes $\mathrm{p}_{\mathrm{i}}$ can now be subtracted from the set of $q_{j}$ where $q_{k}=p_{i}$. Giving the expression $n$ $=1=\mathrm{q}_{\mathrm{m}+1} \mathrm{q}_{\mathrm{m}+2} \ldots \mathrm{q}_{\mathrm{k}}$ and since $\mathrm{q}_{\mathrm{j}}>1$ there stands an inequality with the equation for $\mathrm{n}$; concluding that $\mathrm{p}_{\mathrm{m}}=$ $\mathrm{q}_{\mathrm{k}}$. This now shows that there is a complete uniqueness to the prime number divisors and so proving the Fundamental Theorem of Arithmetic (Burton, 2006).

There has been some confusion throughout the history of prime number theory with disagreements between number theorists. The simplest of these is the question of whether 0 and 1 are prime numbers. While the general population of modern mathematicians accept that 0 and 1 are not prime numbers some earlier mathematicians believed that 1 was a prime number in their ground breaking proofs and conjectures. For example, Goldbach (Burton, 2006) considered 1 as a prime number in his famous conjecture about prime numbers detailed later. As defined earlier, a prime number by definition is an integer that has only the two divisors while composite numbers have a finite number 
of divisors other than one and itself. From this definition it can be seen that both 0 and 1 are not prime numbers due to the fact that 1 has only the one divisor and 0 having infinitely many. If 1 was to be raised to the category of prime numbers then additional qualifications would be compulsory for the Fundamental Theorem of Arithmetic. One such addition would be that the natural number one can be represented by a unique product of prime numbers which leaves this unique list as; 1 .

Measure of primes: how many primes are there? Given that the description of a prime number is to some degree accepted and satisfied by the Fundamental Theorem of Arithmetic another question was then posed as to how many prime numbers are there altogether. The following quote is from Euclid:

"Prime numbers are more than any assigned multitude of prime numbers."

The proposition 20 of book IX of Euclid's Elements states that prime numbers are infinitely many (Siu and Tzanakis, 2004).

Euclid of Alexandria born in Greece around 330BC wrote a thirteen volume mathematical set of books, the Elements. Amid a number of the mathematics theorems that feature throughout the thirteen books is the theorem that there are infinitely many prime numbers. The proof is rather involved representing integers as line segments and measurement as the divisibility of those segments. One could assume that this might be because Euclid was attempting to demonstrate the real life aspect of numbers such as physical quantities that can be measured. The proposal that prime numbers are infinitely many can be made from a few simple statements. If you have a finite list of the first primes $\left(\mathrm{p}_{1}, \mathrm{p}_{2}, \ldots, \mathrm{p}_{\mathrm{k}}\right)$ and then multiply them together and add one you will either get a composite or prime integer. If it is prime then it is a new prime not from the original list. If it composite then its divisors will not be the same as the original multiplication of $n$ primes and so the list is incomplete. The following proof is a summary of what Euclid provided in his book and is worth repeating here just to understand the nature of proofs at that time and the fact that mathematicians were critically considering primes.

Proof: Euclid denoted A, B and C to represent his prime numbers. Then taking the least number now represented by DE measured by $\mathrm{A}, \mathrm{B}$ and $\mathrm{C}$ and adding the unit, measure one, DF to DE. Now, EF can either be a prime number or not a prime. Therefore by allowing $\mathrm{EF}$ to be prime the prime number list becomes A, B, C and EF which are more than our original list. Therefore the list of prime numbers was shown to be incomplete.

Next, let EF not be a prime number and as a result of the Fundamental Theorem of Arithmetic, there is some prime number that measures $\mathrm{EF}$, that will be represented by $\mathrm{G}$. There are now two alternative choices for $\mathrm{G}$, either $\mathrm{G}$ is the same measure as A, B, or $\mathrm{C}$ or it is not alike. Using the first case, where $\mathrm{G}$ is the same as $\mathrm{A}, \mathrm{B}$, or $\mathrm{C}$ it can be seen that $\mathrm{A}, \mathrm{B}$ and $\mathrm{C}$ all measure $\mathrm{DE}$, therefore $\mathrm{G}$ is also a measure of $\mathrm{DE}$. However, by knowing $G$ measures EF, G must also measure the unit DF, which is impossible. Therefore, $G$ is not the same measure as $\mathrm{A}, \mathrm{B}$, or $\mathrm{C}$ and by definition, $\mathrm{G}$ is a prime. For this reason, prime numbers $\mathrm{A}, \mathrm{B}, \mathrm{C}$, are joined by $G$ which is found to be more than our original list of primes. Therefore, prime numbers are more than any assigned multitude of prime numbers (Starbird, 2008).

Euclid's proof of infinite primes is accepted throughout modern mathematics however there has been more than one widely accepted proof. Having already shown the proof for infinite primes the next will be a brief comparison between a few of the highly regarded proofs.

In the 1700 s Euler used the prime series $\sum \frac{1}{\mathrm{p}}$ (where $p$ is prime numbers) to show that the series diverges, furthermore showing that primes are infinitely many. If we take a simple look into Euler's proof we take any integer and break it up into its unique set of prime divisors. $\left(n=p_{1}^{n_{1}} p_{2}^{n_{2}} \ldots p_{k}^{n_{k}}\right)$ From the list of integers of this form it can be shown that if the set of prime numbers is finite then the series of their reciprocals as Euler showed converges and so contradicts $\sum \frac{1}{n}$ which we know diverges. Thus proving the list of prime numbers is infinite. The proof is more involved with derivations on how Euler proved the series convergence then the above explanation and numerous writings have been produced with comprehensive accounts but a simplified view gives an easier explanation for all mathematicians and so can motivate into reading deeper. A more detailed look into this proof and its connection with the zeta function can be found from (Leonhard Euler: His Life. The Man and his Works, Gautschi, 2008). Used a counting proof that could also show that the prime series diverges but through different methods. Fürstenberg (1955) showed that the set of prime numbers is infinite by considering the implications of a set of finite primes through the use of topology. All four cases use the proof by contradiction, reductio ad absurdum (Siu and Tzanakis, 2004). 


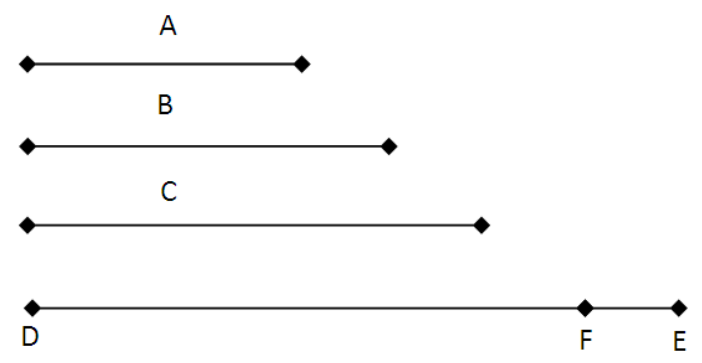

Fig. 1: A visual representation of Euclid's infinite prime proof

$23-5-7--11-13--17-19--23--29-31--2-37--41-43-47-$

Fig. 2: An example of the Sieve of Eratosthenes for $n=50$

Distribution of prime amongst numbers: The distribution of prime numbers throughout a list of integers leads to the emergence of many unanswered and partially answered questions (Stein and Mazur, 2007). The prime list is infinite with various almost random gaps in-between each one and so becomes a great mostly unsolvable riddle. This creates a great mystery surrounding the questions about prime numbers and so supplies great motivation to all mathematicians new and learned in the subject. History has shown that without a great deal of advertisement the challenges of prime numbers have been treated with determination instead of defeat. The first and most important question that may be the simplest is which of the numbers in a given list of integers are primes? As the research on prime numbers deepened many questions about them naturally followed. For example, when given a specific number $\mathrm{N}$ does a simple formula exist to determine the next prime number or the prime number before it? Also, whether patterns exist within the distribution of primes and whether it would continue through the set of integers. Other questions were also considered such as whether prime numbers share certain characteristics and discernable patterns. Indeed all of these questions have been examined by the greatest minds in mathematics yet most still remain largely unanswered.

Taking a look at the first question and its history leads to the discovery of the Sieve of Eratosthenes (Fig. 1 and 2). The director of the famous Library of Alexandria was the founder of the Sieve of Eratosthenes. Eratosthenes of Cyrene (276BC-194 BC) was a scholarly man who provided insight into many academic topics. He was given the nickname Beta and Pentathlos (Pent athlete) because he was considered second in almost all fields of intellect at the time
(Bales, 2008). He was a gifted academic who knew of the proof that if any primes less than the square root of the given integer is not a divisor of that integer then the integer is a prime number. Using this he devised a way to find all the prime numbers below a given integer.

Proof: Given a composite integer $\mathrm{q}=\mathrm{ab}$ where $\mathrm{a} \leq \mathrm{b}$ then $\mathrm{a}^{2} \leq \mathrm{ab}=\mathrm{q}$. From this it can be seen that $\mathrm{a} \leq \sqrt{\mathrm{q}}$ where $\mathrm{p} \mid$ a for some prime $\mathrm{p}(\because$ all integers are divisible by a prime number from the Fundamental Theorem of Arithmetic). Since a $\mid \mathrm{q}$ then it stands that $\mathrm{p} \mid \mathrm{q}$ where $\mathrm{p} \leq$ $\sqrt{\mathrm{q}}(\because \mathrm{a} \leq \sqrt{\mathrm{q}})$. If $\mathrm{q}$ is a prime number then its factorisation can only be itself and one $(a, b=q, 1)$. Therefore showing that there will be no prime (where $p$ $\leq \sqrt{\mathrm{q}}$ ) that is a divisor of $\mathrm{q}$ (Burton, 2006).

Using the above proof the Sieve of Eratosthenes was created to show that given a list of integers up to a given value $\mathrm{n}$ one can remove any composite integers by taking out any multiples of $p$ where $p$ is all primes $\leq$ $\sqrt{\mathrm{n}}$. The straightforward model of this up to the integer 50 is given below.

This answers the question of which numbers in a given list of integers are prime numbers but requires extremely large computations for large values of $n$. Due to the growing demand on discovering the biggest prime numbers and their uses, this design becomes rather cumbersome because of the almost impossibly long calculation one must make. Therefore to answer the question fully, another simpler way had to be discovered.

The Prime Number Theorem was devised from a colourful history to obtain a close to complete answer to the question of distribution of primes. The Prime Number Theorem asserts that:

$\lim _{x \rightarrow \infty} \pi(x) / \frac{x}{\log (x)}=1$

where, $\pi(x)$ is the number of prime numbers less than a given integer $(x>1)$. This Prime Number Theorem was first conjectured by both Gauss and Legendre separately. The first announcement that there was an elementary proof came in 1948 from Paul Erdos and Atle Selberg who used properties of logarithm functions to find the simple proof. Following this publication there was a bitter dispute involving both mathematicians that lasted many years. The quarrel began over the question of a joint paper involving the release of The Prime number Theorem. Why or even how the dispute began is not important in a mathematical sense but the implications on both the real life of mathematics and the mathematics society can be 


\section{J. Math. \& Stat., 7 (4): 262-269, 2011}

significant to aspiring mathematicians. One view on this takes a general look that mathematics involves more than just numbers and black boards but also politics, passion and dedication. It is no longer just a boring look at historical numbers and theories but a real life feel to the story behind the work. After the dispute Erdos and Selberg received the Cole prize (1952) and Fields Medal (1950) respectively and Erdos had written over 1500 published papers in his lifetime (Goldfeld, 2004). Erdos dedicated his life to furthering our understanding in numerous mathematics topics. One could make the assumption that because of the dispute over The Prime Number Theorem both mathematicians dedicated their work to proving themselves to one another and the mathematics community. A further look into this dispute can show that it gives an interesting twist to future mathematicians who are looking over the history of their topic. It shows that mathematics is not someone in the back corner of the library reading a book of numbers but has real life motivation that can appeal to a wider range of mathematicians especially at a younger age. With a wider collection of mathematicians a deeper understanding can be reached within all fields of mathematics and so controversial instances in history can actually help both the mathematicians included and future mathematicians recounting the history.

Prime questions: The consideration of prime numbers throughout the list of integers promptly leads to a multitude of questions. Many of these questions are in relation to the distribution of primes throughout the infinite list of integers. The twin prime conjecture is the first problem from a large list of unanswered questions. It looks at the distribution of prime numbers such as 11 and 13 that are separated by a single even integer. The question is simple, do the pairs of prime numbers continue on indefinitely? The question has been answered in a sense through experimental computation to extremely large numbers, an example of this (Stein and Mazur, 2007; Oladejo and Adetunde, 2009):

'There are 1,177,209,242,304 such pairs less than $1,000,000,000,000,000$ '

While this may be an experimental proof to an extremely large number the question of whether they continue on indefinitely has most certainly not been mathematically proven. Further questions concerning the separation between primes are also unanswered such as are there an infinite many prime number separated by 3 composite numbers.
The Goldbach Conjecture is another unproven question that lies in the realm of prime numbers. In the year of 1742, Christian Goldbach sent a letter to a fellow prime number theorist, Leonhard Euler to express a pattern he had discovered. He surmised that every even integer can be broken into the summation of two prime numbers or 1 . Holdback himself considered 1 as a prime number within his work. Moreover, Goldbach conjectured that all odd integers above 5 can be expressed as a sum of 3 prime numbers (Burton, 2006).

This conjecture has had many attempted proofs but none as of yet to be accepted by the mathematics community. It has however been strongly and persistently tested within very large parameters using computer analysis.

Certain types of prime numbers have also been discovered in attempts to discover the truth behind prime numbers. A short list of several of these famous primes include Fermat primes of the form $2 p+1$, Fibonacci primes which are of the form of Fibonacci integers, Sophie Germaine primes which are of the paired form $p$ and $2 p+1$ and last for this list but certainly not for the complete list are the Mersenne primes.

Marin Mersenne had primes of the form $2^{n}-1$ named after him for his work on providing his conjecture and the list of those primes. It was later disproven for the list of all complete values of $n$. A fact that paved the way for the theorem that if there is a Mersenne prime then the integer $n$ is prime if $n$ is derived from $2^{\mathrm{n}}-1$. Sadly for Mersenne's conjecture this is not always the contrasting case (Starbird, 2008).

Finally but certainly not least a look into the Riemann Zeta Function and its application to prime numbers will be considered. This may be giving justice to the saying, save the best to last because in the study of prime numbers the zeta function is in the highest regard. In 1859, Riemann discovered there was a correlation between the zero values of the zeta function (1b) and prime numbers.

$$
\zeta(s)=\sum_{n=1}^{\infty} \frac{1}{n^{s}}
$$

The above equation converges for $\mathfrak{R}(\mathrm{s})>1$ where $\mathrm{s}$ is defined as a complex number and this can be shown to extend over the entire complex plane through analytic continuation. Using this function Riemann was able to show that all non trivial zero values for the Zeta function lie on the line $\mathfrak{R}(\mathrm{s})=\frac{1}{2}$ which is known as the critical line with only one singularity (Fekih-ahmed, 2011). 
This is known as the Riemann conjecture and is famously still unproven. The connection to prime numbers comes from both Riemann's conjecture and Leonhard Euler's discovery:

$$
\varsigma(\mathrm{s})=\prod \frac{1}{1-\mathrm{p}^{-\mathrm{s}}}
$$

where, p represents prime numbers.

Applications of primes: Why prime numbers are so important and what they can be used for has been better answered over the last 40 years in comparison to the long history of prime numbers. Prime numbers are unique and are considered to have a streak of randomness about them so it is unsurprising that the large attention they receive creates opportunities for real life applications. There are now various applications for prime theorems that have real life significance. Encryption is one of the foremost applications of prime numbers. It uses prime numbers and the almost unworkable job of factorising exceptionally large numbers to encrypt, which is to jumble important information such as credit card numbers into unusable information for any other then the intended receiver. Prime numbers are used to keep important information safe which reveals just how important an application it is.

An indirect application of prime numbers was discovered and used by musicians throughout history. Certain musicians enjoyed the idea of the unique properties of prime numbers to give their music a sense of freedom from the rules governing traditional music. Olivier Messiaen was a composer who used prime numbers throughout his music for this reason (Starbird, 2008).

Another real life application can be seen from nature itself. There are certain insects whose very lives revolve around the use of prime numbers. An insect known as the Cicada uses prime numbers in their existence by living underground for a prime number of years and emerging as an adult at the conclusion of their time. Prime numbers have a special trait that comes in handy for their insect friends. Prime numbers do not interact with each other very often. For the Cicada's instance, they appear above ground every 13 or 17 years. These two prime numbers only interact with each other, namely, $13 \times 17=221$. Consequently the two groups of Cicadas only appear on the surface at the same time every 221 years. A simple numerical counter example of this is 6 and 4 which are composite numbers. 6 and 4 interact much earlier than their given $6 \times 4=24$ because they both interact at 12 . This aids the
Cicadas by making it difficult for interbreeding. It also creates difficulty with any predator hoping to catch the Cicadas unawares as they appear from underground because the predator's life cycles interact with fewer occurrences. This is a case where the unique qualities of prime numbers lend a hand to those in nature stuck in the dirt (Goles et al., 2000).

Prime numbers are no longer chained to purely mathematical sciences. There has been use of prime numbers within theoretical physics to try and produce a theory that ties together prime numbers with quantum chaos theory. It would seem that both mathematicians and physicists are working together to provide a further understanding of how our universe is governed and are doing it through the help of prime numbers.

An interesting financial incentive for more application of prime numbers is the reward for the search for the largest prime number. The Electronic Frontier Foundation offers financial rewards starting from $\$ 50,000$ and increasing rapidly for the discovery of the largest prime number. While this is not a direct application onto the real world for prime numbers it certainly opens the door for a large motivated audience into the study of prime numbers.

\section{CONCLUSION}

This study has briefly reviewed the history of numbers and more specifically prime numbers and has suggested reasons why the continuation of their study is both necessary and appealing to all. A closer look behind the mathematicians and their work has shown a deeper understanding of the real life involved as passions and politics and reasons why this can be a positive contributor to the understanding of mathematics. In this study, a simple description of what a prime number is and how many primes are there was considered through a detailed description of historical proofs. The second half of the study briefly examined the many prime questions that are yet to be proven. Finally, some real life applications of prime numbers were used to show the importance of prime numbers to our society. As a result, there is a need to renew interest and further investigate primes to better understand such numbers that are the building blocks of our number system and so casually used by all.

\section{REFERENCES}

Al Mijalli, M.H., 2011. Efficient realization of s-box based reduced residue of prime numbers using virtex-5 and virtex-6 FPGAs. Am. J. Applied Sci., 8: 754-757. DOI: 10.3844/ajassp.2011.754.757 
Bales, S.E., 2008. Aristotle's Contribution to Scholarly Communication. University of Tennessee, Knoxville.

Burton, D.M., 2006. Elementary Number Theory. 6th Edn., Tata McGraw-Hill Education, New York, ISBN: 0070616078, pp: 434.

Fürstenberg, H., 1955. On the infinitude of primes. Am. Math. Monthly, 62: 353-353.

Gautschi, W., 2008. Leonhard euler: His life, the man and his works. Soc. Indus. Applied Math. Rev., 50: 3-33.

Goldfeld, D., 2004. The elementary proof of the prime number theorem: An historical perspective.

Goles, E., O. Schulz and M. Markus, 2000. A biological generator of prime numbers. Nonlinear Phenomena Complex Syst., 3: 208-213.

Oladejo, N.K. and I.A. Adetunde, 2009. A numerical test on the Riemann hypothesis with applications. J. Math. Stat., 5: 47-53. DOI: 10.3844/jmssp.2009.47.53
Ore, O., 1988. Number Theory and Its History. 1st Edn., Dover Publications, New York, ISBN: 10: 0486656209, pp: 380.

Ribenboim, P., 1996. The Book of Prime Number Records. 3rd Edn., Springer, New York, ISBN: 0387944575, pp: 541.

Siu, M.K. and C. Tzanakis, 2004. The Role of the History of Mathematics in Mathematics Education. Cyprus Mathematical Society, pp: 363-367.

Starbird, M.L., 2008. The mathematics behind prime numbers. J. Math. Comput. Sci. Scholarship, 1: 1519.

Stein, W. And B. Mazur, 2007. What is Riemann's Hypothesis? Lecture presented at Summer Institute for Mathematics at the University of Washington, DC. 